\title{
RECURSOS EDUCATIVOS PARA UNA AGENDA FEMINISTA DESDE LA DIDÁCTICA DE LAS CIENCIAS SOCIALES
}

\author{
EDUCATIONAL RESOURCES FOR A FEMINIST AGENDA
}

FROM THE DIDACTICS OF SOCIAL SCIENCES

\section{RESUMEN}

La realidad social todavía cuenta con un importante componente discriminatorio y siguen surgiendo amenazas que, desde diferentes sectores, cuestionan y ponen en riesgo la lucha feminista. Esta situación justifica la necesidad de revisar y reflexionar el presente para proyectar los desafíos futuros: qué hacer y cómo hacerlo. Ante el reto de plantear una agenda feminista, este texto llama la atención sobre la importancia de la educación de las nuevas generaciones como palanca de cambio para normalizar la igualdad. Ante libros de texto y materiales que a menudo no transmiten fehacientemente la igualdad entre sexos, que no cuidan el lenguaje sexista o que invisibilizan la aportación histórica de las mujeres, se hace necesario incluir en la agenda propuestas concretas en el ámbito educativo. El objetivo aquí es compartir recursos y procesos diseñados para educar en igualdad a las nuevas generaciones de niños y niñas.

Palabras clave: Recursos educativos, Objetivos de Desarrollo Sostenible, matemagia, ludificación, científicas.

\section{ABSTRACT}

The social reality still has a big discriminatory component and threads that put at risk the feminist struggle keep on appearing from different sectors. This situation justifies the need to think about the present and check it out to put forward the challenges for the future: what to do and how to do it. Facing the task of planning a feminist agenda, this paper draws attention to the importance of new generations education as a driving force for change to normalize gender equality. Facing textbooks and other school materials that often do not transmit faithfully gender equality, do not take care about sexist language and even 
make invisible the historic women contributions, it is necessary to include in the feminist agenda specific proposals for the educational environment. The aim here is to share resources and processes designed to educate on gender equality the next generations of boys and girls.

Keywords: Educational resources, Sustainable Development Goals, mathemagic, gamification, scientific women.

\section{SUMARIO}

1.- Introducción. La educación en la configuración de una agenda feminista. 2.- Educación e igualdad de género en el marco de los Objetivos de Desarrollo Sostenible. 3.- El juego como metodología. Juegos matemágicos. 4.- Un recurso para la visibilidad y la igualdad: la baraja de cartas de África. 5.- La baraja de mujeres científicas.

\section{Introducción. La educación en la configuración de una agenda feminista}

El feminismo como teoría, pero sobre todo como movimiento social, se encuentra en la actualidad en su mejor situación histórica. Desde su nacimiento a finales del siglo $\mathrm{XVIII}$, cuando las conquistas en materia de igualdad jurídica, derechos y libertades de la Revolución Francesa y otras revoluciones liberales dejaron de lado a la mujer, la lucha se ha mantenido vigente y creciente. En su recorrido histórico, como explica Valcárcel (2017), el feminismo ha vivido importantes inflexiones, cada una de las cuales ha estado acompañada de una agenda específica que definía los temas principales de los que la vanguardia del movimiento debía ocuparse. Los acontecimientos de los últimos años -el \#MeToo, la cada vez más decidida intransigencia hacia la más mínima violencia sexual, la demanda de la plenitud de derechos, las exigencias de paridad a todos los niveles, la lucha por la destrucción del techo de cristal y por la desaparición de la brecha salarial, el seguimiento masivo y comprometido de los últimos 8 de marzo, pero también, por otra parte, los crecientes discursos de odio, la presión de sectores religiosos ultraconservadores y la ofensiva de la extrema derecha política- sitúan al feminismo actual ante una nueva y crucial inflexión que necesita, como en ocasiones anteriores, la definición de una agenda que no solo impida el más mínimo retroceso, sino que garantice el avance por el que tanto se ha luchado.

Plantear una agenda feminista es pensar y diseñar qué se quiere conseguir y cómo hacer para conseguirlo. La planificación debe afectar a diferentes ámbitos como los movimientos sociales, el activismo, la creación artística y literaria, los medios de comunicación o las instituciones, entre otros. De entre todos los territorios donde debe operar la agenda feminista, la educación puede y debe ser uno a tener muy en cuenta y al que dedicar espe- 
cial atención, precisamente por su potencial. Se trata de uno de los principales agentes de socialización, pues actúa sobre las niñas y niños ya desde sus primeros años. Además, se mantiene durante las etapas más importantes en la formación cognitiva y socioafectiva de las personas, como son la infancia y la adolescencia y, en muchos casos, más allá, durante la formación superior. Por su temprana y prolongada incidencia sobre niñas, niños y jóvenes y por la trascendencia en la configuración de las respuestas conductuales y emocionales que tendrán en el futuro, la educación -como espacio desde el que operar- debe tenerse muy en cuenta en todas las transformaciones sociales $y$, en concreto, en la que persigue una igualdad definitivamente real y plena.

Una revisión de la situación del sistema educativo, desde la perspectiva de la cuestión de género, muestra avances importantes respecto al pasado, pero también muchas cosas todavía por cambiar y mucho margen para la acción. Las últimas leyes estatales, LOE y LOMCE, establecen entre sus principios generales el desarrollo de valores que fomenten la igualdad efectiva entre hombres y mujeres. Un principio que más adelante se articula en forma de objetivo concreto en cada etapa educativa y que concierne también a la formación del profesorado, al consejo escolar, a la inspección educativa o a los materiales curriculares.

Pese a la legislación educativa y su buena intención, como indican Díaz de Greñu y Anguita (2017: 220), la desigualdad subyace en el currículum oculto. Ortega (2018: 13) explica de qué forma la investigación reciente constata que las representaciones del alumnado y profesorado en formación, los currículos y los libros de texto continúan invisibilizando a las mujeres en medio de un discurso marcadamente patriarcal. Ello contribuye a disminuir, o directamente ocultar, la verdadera diversidad de género y a mantener estereotipos e identidades hegemónicas. Para evitarlo quedan algunas acciones importantes a acometer. En lo que se refiere al profesorado, es necesaria su formación específica en materia de género, la inclusión de esta en los planes de estudio universitarios y el trabajo desde modelos epistemológicos y metodológicos que puedan transformar la práctica docente (Ortega, 2018:14).

Por su parte, los materiales curriculares deben ser diseñados con especial atención al relato, al lenguaje y a las imágenes, para no perpetuar modelos androcéntricos, incurrir en discriminaciones ni sugerir desigualdad entre géneros. Como explican y demuestran Gutiérrez e lbáñez (2013:111), los libros escolares en muchos casos todavía muestran tareas o características estereotipadas que, asimiladas por el alumnado, tienen consecuencias importantes en su forma de pensar, interpretar y relacionarse en el futuro. En cuanto a cómo cambiar esta realidad Ortega (2018:13), que específicamente trata sobre la enseñanza de la Historia, lleva a cabo una reflexión que puede extrapolarse al resto de materias del currículo. De hecho, plantea la necesidad de un cambio en el discurso y sugiere abandonar soluciones 
eventuales en forma de anexos específicos, añadidos o complementos meramente testimoniales. Con todo, mientras se hace efectiva la transformación en la práctica docente, en los discursos y en los materiales curriculares, recursos didácticos como los que se presentan en esta comunicación, desarrollados y puestos en práctica por el grupo DIMPA, permiten acercar al alumnado al conocimiento integral de la realidad presente y pasada, con su verdadera diversidad de género, y se constituyen en eficaces herramientas al servicio de una agenda feminista desde la educación.

\section{Educación e igualdad de género en el marco de los Objetivos de Desarrollo Sostenible}

El grupo DIMPA de la Universitat Jaume I de Castelló trabaja en la investigación y en la producción de recursos para el aula, desde la perspectiva de la didáctica de las Ciencias Sociales. Junto con la cultura, el patrimonio o la interculturalidad, la igualdad de género es uno de los ejes a partir de los cuales se entiende el trabajo del grupo y de las profesoras y profesores que lo integran. Con este último, el equipo se suma a trabajar desde la educación para alcanzar con éxito el quinto de los Objetivos de Desarrollo Sostenible planteados por Naciones Unidas en el marco de la Agenda 2030: conseguir la igualdad entre géneros y empoderar a mujeres y niñas como base para la construcción de un mundo pacífico, próspero y sostenible.

El principio de igualdad entre mujeres y hombres consta de legislación específica en España y otros países, figura en declaraciones internacionales y está en el mismo centro de los derechos humanos universales, pero es una realidad que se encuentra muy lejos de ser alcanzada completamente. Está especialmente lejos en algunos contextos donde las condiciones sociales, culturales y económicas, que al fin son absolutamente determinantes en la construcción de un marco favorable para su reivindicación, se muestran adversas. De hecho, en 2014 todavía había más de cincuenta países en todo el mundo que en sus leyes y constituciones no garantizaban la igualdad de género (Naciones Unidas, 2015). A lo largo de las dos últimas décadas ha habido algunos avances a escala global, pero la realidad es que las mujeres y las niñas siguen sufriendo discriminación y violencia en todos los lugares del mundo.

La igualdad de género es el quinto de los Objetivos de Desarrollo Sostenible (ODS) señalados por Naciones Unidas en el marco de la Agenda 2030, una serie de objetivos globales para tratar de eliminar la pobreza, proteger el planeta y garantizar la prosperidad mundial. Alcanzar la igualdad entre los géneros y empoderar a mujeres y niñas es, y así se 
subraya, una base necesaria desde la que construir un mundo pacífico, próspero y sostenible. Si bien este es un objetivo específico, para el que se establecen unas metas concretas, el trabajo por la cuestión igualitaria se puede abordar, además, desde otros Objetivos de Desarrollo Sostenible. El objetivo cuatro, que pretende garantizar una educación inclusiva, equitativa y de calidad para todos, puede ser, según las Naciones Unidas, fundamental para conseguir otros objetivos porque posibilita escapar del ciclo de la pobreza, fomenta la tolerancia, ayuda a crear sociedades más pacíficas y, lo más interesante para este caso, contribuye a reducir las desigualdades y a conseguir la igualdad de género (Naciones Unidas, 2015). Son todavía muchos los países en los que las niñas tienen más dificultades para hacer los estudios primarios, arrastrando así una desventaja que luego se traduce en falta de capacitación y de oportunidades laborales. Además de la escolarización universal que reclama el ODS, a través de la educación se puede formar en igualdad y se puede contribuir activamente a erradicar aquellos factores que terminan por justificar, fomentar o sostener la desigualdad entre mujeres y hombres, llegando a anularla o, al menos, minimizarla en el futuro.

Convencidos del papel clave que debe tener la educación en la transformación de la sociedad, en la consecución de una verdadera igualdad, y comprometidos con el trabajo por los ODS desde las aulas, el grupo DIMPA trabaja en la investigación y el desarrollo de estrategias y recursos como los que aquí se exponen. Unos materiales que aspiran a visibilizar y dar a conocer las grandes aportaciones de la mujer y que pretenden servir como estrategia y recordatorio -porque hoy en día todavía es imprescindible- de la necesidad de situar en el mismo plano a mujeres y hombres en cualquier disciplina o ámbito vital.

\section{El juego como metodología. Juegos matemágicos}

Como se ha dicho anteriormente, la igualdad de mujeres y hombres debe abordarse directamente en la escuela. Se debe explicar, mostrar y demostrar al alumnado este estado de paridad sin ambages, con la claridad y normalidad que, precisamente, se aspira a conseguir. Pero además, se puede aprovechar el margen que deja la actividad cotidiana para continuar con esta pedagogía de equidad de una forma menos sistemática y evidente, que no menos importante, a través de medios que pueden complementar el discurso y hacer llegar el mensaje tácitamente. El juego es un claro ejemplo de este tipo de recursos. Su potencial como estrategia de enseñanza y aprendizaje está bien justificado y es ampliamente aceptado por los profesionales del mundo de la educación. De hecho, su presencia es bastante habitual en los primeros niveles de enseñanza y es sólo con el paso de los cursos cuando se hace más y más excepcional, hasta acabar reducido a alguna materia o mera solución esporádica para 
trabajar un contenido específico (Bernabeu y Goldstein, 2009: 54). No debería ser así y, por todo lo que pueden aportar los recursos lúdicos como complemento de las actividades más o menos convencionales, el grupo de investigación DIMPA defiende su presencia recurrente desde la enseñanza infantil hasta el nivel universitario. Precisamente: «[...] el juego trata de un acto agradable, con grandes dosis de plasticidad y libertad, un acto de expresión y expansión de la personalidad humana, y por tanto un ejercicio importante a cualquier edad» (Ramiro, Selma y Prades, 2015: 1030).

En general, el juego es también una forma de aprendizaje y resulta fundamental para adquirir destrezas, establecer vínculos o aprender comportamientos adaptativos (Ramiro, Selma y Prades, 2015: 1030). En el aula, en concreto, facilita la adquisición de conocimientos, dinamiza las clases, motiva y aumenta el interés del alumnado, fomenta la cohesión y la solidaridad, favorece la inteligencia emocional y la autoestima, aumenta la responsabilidad y exige actitudes tolerantes y respetuosas (Bernabeu y Goldstein, 2009: 54). A todo esto, cabe añadir que el juego proporciona un aprendizaje subliminal que resulta absolutamente fundamental: las alumnas y alumnos juegan, se divierten, disfrutan $y$, a menudo sin ser conscientes, aprenden. Este planteamiento alrededor del juego y sus posibilidades didácticas se ha tenido muy presente en los sucesivos proyectos desarrollados por el grupo de investigación DIMPA lo largo de los últimos años. Del mismo modo, la apuesta por una metodología lúdica se ha materializado en la producción de recursos en forma de juego con los que trabajar en las aulas temas clave como el patrimonio, la interculturalidad o la igualdad de género.

Además de las barajas de cartas en las que se centra fundamentalmente este texto, en 2014 se desarrollaron Los juegos de la pizza, un conjunto de seis juegos -Personajes de la Península lbérica; Personajes del resto del mundo; Juego de las creencias; $\dot{\partial} T e$ animas a celebrarlo?; ¿Qué paisaje apadrinas?; ¿Qué instrumento prefieres?- con los que se pretende «contribuir a difundir la riqueza que representa una visión del mundo intercultural y crear vínculos positivos y actitudes favorables hacia esta realidad» (Ramiro y Gil, 2017: 58). Más recientemente, en 2017, se han publicado los Juegos interculturales de mesa, una selección de doce entretenimientos originarios de diferentes lugares y culturas del mundo -tres de América, dos de Oceanía, dos de África, tres de Asia y dos de Europa- con los que poder aproximarse a otros contextos culturales a través de la forma de pasar el tiempo libre y divertirse de su gente. El objetivo fundamental es generar un mundo compartido, un medio de conocimiento y aproximación intercultural que pueda favorecer la convivencia y la sensibilidad mutua.

Los recursos didácticos que se presentan en esta comunicación, especialmente la baraja de mujeres científicas, se prestan a innumerables juegos, desde los más convencionales 
a otros más novedosos e incluso a los que pudieran ser inventados por aquellas y aquellos que los usen. Pero en concreto, el grupo DIMPA propone una serie de juegos matemágicos que, por su capacidad de sorprender y por su relativa facilidad de ejecución, suponen una solución muy efectiva. Sherard (1998) habla de la matemagia como un híbrido que combina matemáticas y magia, trucos que funcionan porque están basados en determinados aspectos matemáticos. El empleo de juegos matemágicos puede aportar muchas cosas interesantes como recurso para trabajar contenidos curriculares, con el componente añadido en este caso de la diversidad de género presente en la ciencia.

Se han señalado algunos puntos de contacto entre las dos áreas que se aúnan en la matemagia. Aguado (2017: 9) explica que «un mago muestra un hecho sorprendente, inexplicable, mientras que un matemático explica hechos misteriosos que, en muchas ocasiones, podrían llegar a parecer mágicos». El origen de esta relación de contacto entre las matemáticas y lo mágico se remonta muy atrás en el tiempo, pero se suele situar al divulgador científico Martin Gardner como referente de la matemagia moderna gracias a los juegos publicados en la revista Scientific American entre 1957 y 1981 y otras de sus publicaciones.

Dentro del aula, se puede emplear la matemagia como recurso educativo, realizando juegos o ilusiones ante el alumnado con los contenidos que se necesita trabajar. También se les puede involucrar con prácticas que ellos y ellas puedan llevar a cabo, desarrollando la creatividad, la autoestima, el espíritu crítico, la concentración, la coordinación o la imaginación entre otras (Aguado, 2017: 9-10). Diversos autores especialistas en la materia han apuntado los beneficios que se pueden lograr. Ramiro (2017: 56) habla de alegría, imaginación, creatividad y convivencia. Según Aguado (2017: 9), los juegos fascinan y atraen la atención de los estudiantes, les saca de la rutina, combaten el aburrimiento y fomentan el espíritu crítico e imaginativo, aunque el autor se refiere a la aplicación de la magia en general al proceso de aprendizaje. Por su parte, Ruiz (2013: 36) subraya la capacidad que tiene la magia, utilizada en el aula, para estimular la atención, despertar el interés por aprender, conseguir mayor impacto a nivel psicológico y hacer que lo aprendido se recuerde por más tiempo.

\section{Un recurso para la visibilidad y la igualdad: la baraja de cartas de África}

Centrándonos en los recursos para visibilizar la igualdad de género del grupo DIMPA, cabe señalar, en primer lugar, que este objetivo ha ido ganando protagonismo hasta convertirse en fin fundamental de algunas investigaciones y en elemento principal de varios de los materiales producidos. En los ya citados Juegos de la pizza (2014) el objetivo del proyecto 
apuntaba más hacia una diversidad patrimonial y cultural, pero la preocupación por la desigualdad entre géneros se hizo claramente patente puesto que dos de los juegos terminaron por convertirse en denuncia de un estado de cosas tan injusto como lamentablemente real. Para los juegos Personajes de la península ibérica y Personajes del resto del mundo, se contó con la colaboración de una veintena de profesoras y profesores de la asignatura de Ciencias Sociales de diferentes institutos del País Valenciano para seleccionar a las personalidades que más aparecen en los libros de texto. El resultado fue un recurso que servía a la vez tanto para trabajar la asignatura lúdicamente, como para criticar su contenido, porque la casi totalidad de personajes que aparecen son de género masculino (Ramiro y Gil, 2017: 59). Es verdad que históricamente la mujer ha sido relegada a un plano inferior, muchas veces borrada, y su rol en la política, la sociedad, la ciencia o el arte ha sido minimizado. Pero frente a esto, la crítica que aporta este juego no hace sino pedir un esfuerzo y un compromiso para evitar perpetuar esta minimización y dar visibilidad, también histórica, a la mujer en los materiales didácticos. Las investigaciones y recursos posteriores del grupo fueron en esta dirección.

En los Juegos de la pizza ya se dejaba ver un interés por África, algunos de sus paisajes, de sus tradiciones o de sus figuras destacadas aparecen en diferentes juegos. De esta atención inicial hacia el continente vecino y de la constatación compartida con algunas ONG, como llewasi -especialmente ocupada en la defensa y promoción de los derechos de niñas y niños en países de América Latina y África-, de la falta de materiales didácticos para trabajar su realidad, es de donde surge el proyecto "África, tan lejos y tan cerca» desarrollado a lo largo de 2016. Detrás de este proyecto estaba el objetivo de dar a conocer África, su patrimonio, tradiciones, historia y personalidades, toda una riqueza cultural que habitualmente queda ensombrecida por las constantes guerras, el hambre, las miserias o los prejuicios.

En el proyecto sobre África la reflexión en torno a la igualdad de género cobró un papel más destacado. Enfocando el problema desde el ámbito de la educación, se puede observar que según el centro de datos del Instituto de Estadística de la UNESCO, entre 2000 y 2012 el porcentaje de niñas y niños en edad de asistir a la escuela primaria que no estaban escolarizados había disminuido a la mitad, pero todavía era del $22 \%$ en el África Subsahariana (Naciones Unidas, 2015). Se trata de la región con mayor número de niñas y niños sin escolarizar de todo el mundo. Las niñas tienen más dificultades para matricularse en la escuela primaria o para continuar con la secundaria, asumiendo una dificultad para su desarrollo social y personal respecto a los niños. De hecho, son muchos los países que están lejos de la paridad en escolarización, fundamentalmente porque las niñas tienen que hacer frente a desventajas y obstáculos como el rol tradicionalmente reservado para la mujer, el coste económico de la educación, la amenaza de la prostitución, la maternidad precoz, el 
matrimonio prematuro, el trabajo infantil o la distancia en las escuelas (Iversen y Nyamakanga, 2012: 25-33).

Contra esta situación de desigualdad, desventaja e inferioridad de las niñas africanas hay que luchar y sumar desde todos los frentes y en esta línea se sitúa uno de los recursos producidos por el grupo DIMPA dentro del proyecto mencionado. La educación es un derecho humano universal, niñas y niños deben tener las mismas oportunidades para acceder a ella, de modo que no se anulen o se corten trayectorias vitales, dado que quién sabe dónde pueden llegar o qué pueden aportar en el futuro. La baraja África, tan cerca y tan lejos, publicada en 2016, muestra con total paridad en sus cartas a cincuentaidós personajes que han tenido una relevancia especial a lo largo de la historia de este continente. El hecho de que se muestren tantas mujeres como hombres por su aportación trascendente, y más en un territorio con unos condicionantes tan delicados alrededor del género, sirve para dar visibilidad al sexo femenino, pero también para recordar que cualquiera, sin distinción, puede estar llamado a hacer grandes logros a lo largo de su camino personal, que debe empezar por una educación igualitaria e irrenunciable.

La baraja de personajes africanos está formada por los cuatro palos de diamantes, corazones, tréboles y picas. Con ella se puede jugar a cualquier juego de cartas o bien se pueden hacer juegos matemágicos, como los que se explican en un cuaderno complementario que también se va a publicar, tratándose de trucos automáticos basados en propiedades 0 fundamentos numéricos fáciles de hacer y sorprendentes por su resultado. La mitad de la baraja, dos de los palos, está dedicada a veintiséis mujeres seleccionadas después de un intenso trabajo de investigación y documentación. Las particularidades del continente y su historia apuntaban hacia la dificultad de conseguir el mismo número de hombres y mujeres, pero al fin la recopilación fue amplia y en la baraja no pueden estar, por límite de cartas, todas aquellas que lo merecerían. En el diseño se advierte la intención de distribuir a los personajes entre todas las épocas, profesiones y zonas del continente, priorizando el criterio de relevancia.

El resultado final es una baraja que, en cuanto a los personajes femeninos, muestra a las guerreras Amina (Nigeria) y Kempa Vita (Congo); la sultana Bata del Bambara de Adal (Etiopía); las faraones de Egipto Cleopatra VI y Hatshepsut; las reinas Kahina (Mauritania), Makeda de Saba (Etiopía), Nefertiti (Etiopía), Ngola Ana Nzinga Mbande (Angola), Ranavalona (malgache), Kandake Shanakdakheto (Etiopía) y Yaa Asantewaa (Ghana); la princesa Charlene (Zimbawe); las políticas Graça Machel (Mozambique) y Wangari Maathai (Kenia); la empresaria Aminata Traoré (Mali); la periodista Caddy Adsubia (Congo); la modelo Waris Dirie (Somalia); la actriz Charlize Theron (Sudáfrica); la arquitecta Denise Scot Brown (Zambia); la científica Rose Dieng-Kuntz (Senegal); la defensora de los derechos humanos Funmi- 
layo Ransome-Kuti (Nigeria); la escritora Nadine Gordimer (Sudáfrica); la alquimista María la Judía (Egipto); la líder espiritual Nehanda (Zimbawe) y la cantante Vanessa Hau Mdee (Tanzania). Profesiones y dedicaciones muy diferentes, algunas convencionalmente ligadas a los hombres, de ciudadanas de diecisiete países enmarcadas en una cronología que abarca más de tres milenios para destruir estereotipos, dar visibilidad y reclamar oportunidades para que las niñas de hoy puedan llegar a ser naipes en el futuro.

La estructura de las cartas es la misma en todos los casos y trata de concentrar la atención del jugador o la jugadora en la imagen de cada personaje, que en grande y en el centro ocupa la mayor parte del espacio, y en su nombre, que figura a la derecha en letras bien visibles. En la parte superior izquierda, como es habitual, se encuentra el valor y el palo. Bajo de la representación gráfica aparecen los lugares y fechas de nacimiento y muerte, si se da el caso, y una breve reseña biográfica. El juego se completa con dos cartas de comodines que dan información concentrada del Imperio egipcio y el Imperio bantú, dos de los imperios con más extensión cronológica y más importancia histórica del continente africano.

La baraja África, tan cerca y tan lejos es un material único, porque no se puede encontrar ningún otro juego de este tipo que se ocupe monográficamente de personajes africanos y porque lo hace desde una perspectiva comprometida con una igualdad de género que se quiere reivindicar para África y para cualquier lugar del mundo. Esta baraja no quiere encerrarse en las escuelas e institutos, se puede usar igualmente fuera de las aulas y está abierta a todo el mundo para promover así el conocimiento de África y su historia a través de los protagonistas, que han sido mujeres y hombres por igual. Lo importante no es donde se utiliza, sino por qué hay que utilizarla y con qué finalidad: evidenciar la relevancia del papel y las aportaciones de las mujeres, ser críticos con un relato histórico demasiado masculinizado, concienciar y conseguir una igualdad necesaria.

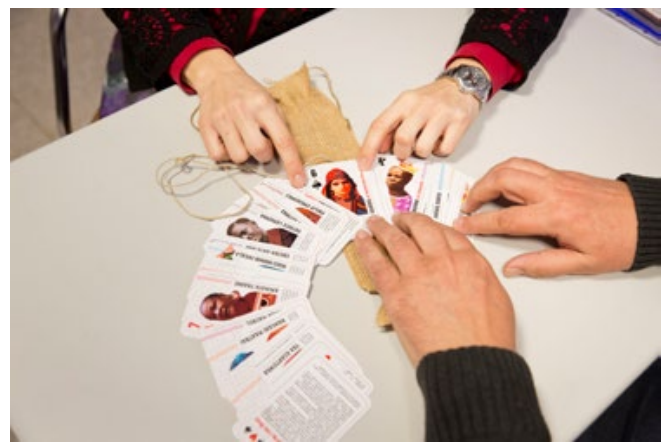

Baraja de cartas sobre África

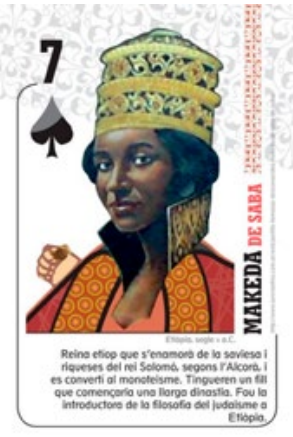

Ejemplo de carta 


\section{La baraja de mujeres científicas}

En 2017 el grupo DIMPA publicó una segunda baraja, en este caso 50 segles de dones científiques per a fer-se visibles, con cincuenta y dos mujeres relevantes de la historia de la ciencia que pudieran reivindicar su valioso y a menudo olvidado papel en este campo. Un recurso concebido para evidenciar la presencia e importancia de las mujeres en la ciencia, cuyo desarrollo histórico se transmite como construido casi exclusivamente por hombres. La baraja, con la carga simbólica que aportan los personajes, cuestiona este relato a la vez que se convierte en medio para ludificar el aprendizaje a través del desarrollo de unos siempre sorprendentes juegos matemágicos. Cada personaje, en cada naipe, consta de su imagen, lugar y fechas de nacimiento y defunción, así como una breve síntesis que explica su importancia.

Si se piensa o se pregunta sobre mujeres científicas, no se suele obtener más que una corta enumeración encabezada por Marie Curie y, con un poco de suerte, de Hipatia. Sin embargo, la historia de la ciencia no se podría hacer sin su trabajo, aunque la inmensa mayoría de sus nombres han pasado al olvido, se han silenciado o borrado conscientemente.

El período investigado abarca desde el siglo XXVIII a. C. hasta la actualidad, con la única limitación de que ninguna de las científicas viva a día de hoy. Evidentemente, es imposible enumerar a todas las mujeres que han destacado durante todos estos siglos y es evidente que cualquier selección nunca será unánime. Sin embargo, se advierte que la baraja intenta respetar un cierto equilibrio entre las diferentes ramas de la ciencia así como una variada distribución geográfica y cronológica.

Según se explica en los créditos de la propia baraja de cartas, la recopilación de mujeres científicas no estuvo exenta de dificultades. La falta de documentación accesible sobre el mundo oriental y la escasa presencia de la mujer en estos ámbitos hasta épocas muy recientes, supusieron un problema para el objetivo propuesto. Igualmente, la gran dificultad en conseguir las imágenes y la información biográfica, ha marcado el resultado. La localización y selección de las mujeres que conforman esta baraja ha contado con el apoyo de un nutrido grupo de colaboradores y colaboradoras que, sin duda, suponen un aval en cuanto a la validez y rigurosidad del criterio para su elección: Ángel Ruiz, presidente del Comité Interamericano de Matemáticas (Universidad de Costa Rica), Josep Ramon BertomeuSánchez, director del Instituto de Historia de la Medicina y de la Ciencia (Universitat de València), Carmen Magallón Portolés, directora de la Fundación Seminario de Investigación para Paz (Centro Pignatelli de Zaragoza), Marta Macho-Stadler, profesora de Geometría y Topología (Universidad del País Vasco-Euskal Herriko Unibertsitatea), Santiago Fernández, asesor de matemáticas del Berritzegune Nagusia-Bilbao, Amable Pérez, asesor científico del Centro de Formación y Recursos para el profesorado de Castelló de la Plana, Manel Josep 
Traver i Ribes, catedrático de Física y Química del IES San Vicente Ferrer de Algemesí, Mercedes Alcañiz, directora de la Unidad de Igualdad (Universitat Jaume I de Castelló), Montserrat Cabré i Pairet, directora del Área de Igualdad, Conciliación y Responsabilidad Social (Universidad de Cantabria), Ana López-Navajas, asesora del Centro de Formación y Recursos para el profesorado específico de Educación Inclusiva (Conselleria d'Educació de la Generalitat Valenciana), Jordi Solbes Matarredona, profesor Titular de Didáctica de las Ciencias Experimentales (Universitat de València) y Carmel Ferragud Domingo, del Instituto de Historia de la Medicina y de la Ciencia López Piñero (Universitat de València).

El conjunto de cartas se divide en los cuatro palos clásicos de la baraja francesa: rombos o diamantes, corazones, tréboles y picas. Cada uno de ellos consta de trece cartas. En relación a los comodines, hay que decir se han seleccionado dos grupos, y no dos individualidades, que representan las bases científicas generales. Por un lado, la Escuela pitagórica de Crotona y por otro, la Casa de la sabiduría de Bagdad, como representantes del mundo occidental y oriental respectivamente. Habría que dedicar un apartado diferente a las comunidades matemáticas de Mesopotamia y Egipto que se desarrollaron antes del siglo XIX a. C. Allí se originó la matemática como una ciencia práctica para facilitar el cómputo del calendario, la administración de las cosechas, la organización de los trabajos públicos y la cuestación de los impuestos, entre otras cuestiones. Por todas estas razones, esta ciencia se fue convirtiendo también en abstracta.

Se ha configurado una baraja compuesta por los nombres que se citan a continuación, agrupados en los cuatro palos de la baraja sin otro principio que el orden cronológico y la necesaria distribución de trece cartas en cada uno de ellos. Las cartas de corazones cuentan con los nombres más antiguos, mujeres que vivieron entre el Antiguo Egipto y la Edad Media. Merit Ptah (Antiguo Egipto, c.2700 a. C.), primera mujer conocida por su nombre en la historia de la medicina $y$, posiblemente, también la primera mujer nombrada en toda la historia de la ciencia; Enheduanna (Mesopotamia, 2285-2250 a. C.), sacerdotisa, astrónoma y poetisa, escribió los primeros textos atribuibles a una autoría; Tapuputi-Belatikallim (Mesopotamia, c. 1200 a. C.), ostenta la primera consideración de química, fabricante de perfumes y nombrada en una tablilla cuneiforme de la época; Gargi Vachaknavi (India, entre los siglos IX y VI a. C.), filósofa y poetisa, autora de himnos védicos, nombrada en la Upanishad Briha daranyaka; Teano de Crotona (Crotona, siglo Vl a. C.), una de las primeras filósofas occidentales, esposa de Pitágoras de cuya escuela formó parte y llegó a ser incluso maestra; Areté de Cirene (Cirene, 435-366 a. C.), filósofa y maestra de la escuela cirenaica, marcada por el pensamiento socrático; Agnódice (Atenas, siglo IV a. C.), se hizo pasar por hombre para estudiar anatomía y fisiología, ante la prohibición para las mujeres, hasta llegar a ser ginecóloga; Aglaonice (Grecia, siglos II-I a. C.), astrónoma, formó parte de «Las brujas de Tesalia»; 
María la Judía (Alejandría, entre los siglos I-III d. C.), fundadora mitológica de la alquimia, se le han atribuido artilugios para destilar y sublimar, así como el famoso «baño María»; Hipatia (Alejandría, 370-415), maestra y cabeza de la escuela neoplatónica de Alejandría, destacó en matemáticas y astronomía; Fátima de Madrid (Madrid, siglos X-XI), astrónoma andaluza, famosa por sus trabajos de astronomía; Tórtula de Ruggiero (Italia, siglo XI), ginecóloga del primer centro médico medieval sin conexión con la lglesia, fue autora de influyentes trabajos sobre la materia; Hildegarda Von Bingen (Alemania, 1098-1179), abadesa, teóloga, músi$c a$, poetisa y naturalista entre otras ocupaciones, es santa de la iglesia católica.

Las cartas del palo de diamantes muestran a Jacobina Felice de Almania (Florencia, siglo XVI), pertenecía a la minoría de las médicas de París con licencia, se le acabó prohibiendo ejercer; Tarquinia Molza (Módena, 1542-1617), filósofa, cantante, poetisa, compositora y directora, considerada una gran virtuosa de la música; Elisabeth Hevelius (Polonia, 1647-1693), astrónoma considerada madre de la topografía lunar, publicó el mayor catálogo astronómico con 1564 estrellas y sus posiciones; María Casamayor y de la Coma (Zaragoza, 1700-1780), matemática y escritora, única científica española del siglo XVIII de la que se conservan sus libros; Marie-Anne Pierrette Paulze (Francia, 1758-1836), madre de la química moderna, autora de múltiples trabajos sobre composición, procesos y transformación de elementos; Sophie Germain (París, 1776-1831), matemática autodidacta, escribió con seudónimo masculino, es bien conocida por el teorema que lleva su nombre; Mary Anning (Inglaterra, 1799-1847), paleontóloga, a ella corresponde el primer hallazgo del primer esqueleto completo de ictiosaurio; Ada Lovelace (Londres, 1815-1852), matemática, madre de la programación; Florence Nightingale (Florencia 1820-Londres 1910), madre de la enfermería moderna, mejoró el servicio sanitario en el ejército, creó la primera escuela laica de enfermería y primera mujer admitida en la Royal Statistical Society; Marianne North (Inglaterra, 1830-1890), naturalista, conocida por sus dibujos de especies naturales de todo el mundo; Sófia Kovalévskaia (Moscú 1850-Estocolom 1891), matemática y profesora en la Universidad de Estocolmo; Ida Henrietta Hyde (Estados Unidos, 1857-1945), bióloga, fisióloga, primera mujer en doctorarse en Ciencias Naturales; Beatrice Webb (Inglaterra, 1858-1943), socióloga, economista, célebre por acuñar el término «negociación colectiva» y ser cofundadora de la London School of Economics.

Las picas incluyen a científicas nacidas entre la segunda mitad del siglo XIX y los primeros años del siglo XX. Agnes Luise Vilhelmine Pockels (Venecia, 1862-Brunswick, 1935), química autodidacta, investigadora sobre química orgánica y mecánica de medios continuos; María Sklodowska (Varsovia, 1867-Sallanch, Francia, 1934), también conocida como Marie Curie, doble Nobel en Física y Química, pionera en radioactividad, descubrió los rayos X; Lise Meitner (Viena, 1878-Cambridge, 1868), física y profesora, investigó sobre radiactividad y 
fisión nuclear; Inge Lehmann (Copenhague, 1888-1993), sismóloga y geofísica; Margarida Comas i Camps (Menorca, 1892-Exeter, Inglaterra, 1973), bióloga y pedagoga, pionera de nuevas formas de enseñanza de las ciencias; Irene Joliot-Curie (Paris, 1897-1956), hija de Pierre y Marie Curie, fue Nobel de Física en 1935; Katharine Burr Blodgett (EEUU, 1898-1979), primera física doctorada en Cambridge, destacó por sus aportaciones a la química industrial; Margaret Mead (Filadelfia, 1901-Nueva York, 1978), antropóloga; Barbara McClintock (EEUU, 1902-1992), Nobel de Medicina por el descubrimiento de la recombinación genética; María Zambrano (Vélez-Málaga, 1904- Madrid, 1991), filósofa, profesora y ensayista; Isabel Torres Salas (Cuenca, 1905-Granada, 1998), farmacéutica y dietista, investigó sobre el valor nutritivo de los alimentos y la estructura de las vitaminas; Maria Goeppert-Mayer (Katowice, Polonia, 1906-San Diego, EEUU, 1972), Nobel de Física en 1963 por proponer el modelo de capas nucleares y Rachel Carson (EEUU, 1907-1964), bióloga y escritora, inició el movimiento ecologista con sus estudios sobre los daños de los insecticidas en la cadena trófica.

El palo de tréboles contiene mujeres científicas nacidas ya en el siglo XX, época histórica en la que su presencia e importancia aumenta de forma considerable, sobre todo con el avance de la centuria, pero sin llegar a ser comparable a la de los hombres. Simone de Beauvoir (París, 1908-1986), fue una relevante filósofa existencialista, profesora, escritora y figura clave en el movimiento feminista; Rita Levi-Montalcini (Torino 1909-Roma 2012), neuróloga y profesora universitaria, Nobel de Medicina en 1986; Dorothy Crowfoot (El Cairo 1910- Shiptons on Stour, Inglaterra, 1994), química y profesora universitaria, Nobel en su especialidad e 1964; Chien-Shiung Wu (Taicang, China, 1912-Nueva York, 1997), física experta en radiactividad; He Zehui (Suzhou, China, 1914-Pekín, 201 1), física nuclear, es una figura icónica nacional y considerada la «Marie Curie oriental»; Sara Borrell Ruiz (Madrid, 1917-1999), doctora en farmacia y profesora en CSIC, creó la Sociedad Española de Bioquímica; Gertrude Bellion (EEUU, 1918-1999), investigadora médica sobre leucemia y otras enfermedades, Nobel en 1988; Rosalind Elsie Franklin (Kensington 1920-Londres 1958), tuvo un papel destacado en el desarrollo de la biología molecular: el descubrimiento de la estructura del ADN; Stephanie Kwolek (EEUU, 1923-2014), química inventora del Kevlar; Maria Assumpció Català i Poch (Barcelona, 1925-2009), primera mujer en doctorarse en matemáticas por la Universidad de Barcelona y profesora universitaria; Carmina Virgili Rodón (Barcelona, 1927-2014), geóloga especializada en sedimentología y estratigrafía del Triásico y del Pérmico, investigadora del CSIC; Dian Fossey (San Francisco 1932-Ruhengerí, Ruanda, 1985), zoóloga y reconocida conservacionista con los gorilas de Ruanda y Congo; Elinor Ostrom (EEUU, 1933-2012), primera mujer economista galardonada con el Nobel en 2009. 


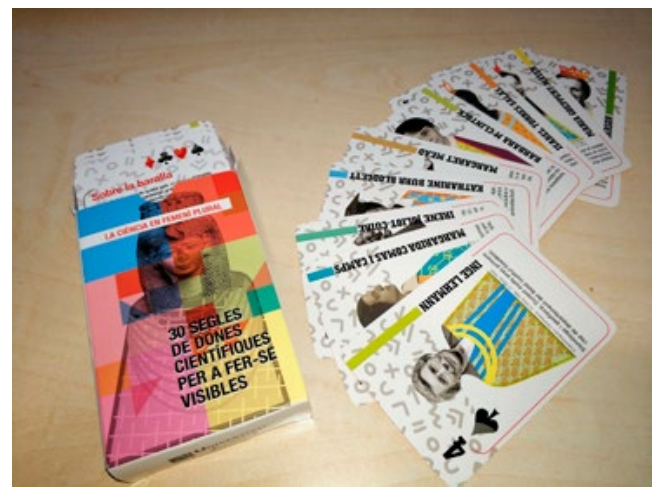

Baraja de mujeres científicas

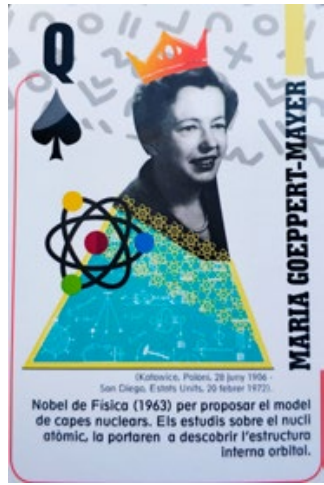

Ejemplo de carta

\section{Bibliografía}

AguAdo, Juan Carlos (2017). «El uso de la magia como recurso docente: el taller de la magia de la Economía» en Teaching and Learning Innovation Journal, №1, pp. 9-13.

AÑó, Raül y Enric Ramiro (2015a). Em coneixes? Joc dels personatges peninsulars. Els jocs de la pizza. Interculturalitat i ciències socials, Navarrés: Babilonia, Asociación Cultural.

AÑó, Raül y Enric Ramiro (2015b). Em coneixes? Joc dels personatges universals. Els jocs de la pizza. Interculturalitat i ciències socials, Navarrés: Babilonia, Asociación Cultural.

Bernabeu, Natalia y Andy Goldstein (2009). Creatividad y aprendizaje. El juego como herramienta pedagógica, Madrid: Narcea.

Díaz de Greñu, Sofía y Rocío Anguita (2017). «Estereotipos del profesorado en torno al género y a la orientación sexual» en Revista Electrónica Interuniversitaria de Formación del Profesorado, №20 (1), pp.219-232.

GutiéRReZ, Prudencia y Patricia lBÁÑEz (2013). «¿Cómo se transmiten los estereotipos culturales y sexistas a través de las imágenes de las TIC en los libros de texto?» en Enseñanza \& Teaching, No31 (1), pp.109-125.

GIL, José Vicente etal. (2018). «El projecte "Àfrica, tan lluny i tan prop": treballant la igualtat de gènere des de la didàctica de les ciències socials» en ALCAÑIz, Mercedes (coord.) (2018). III Congrés Investigació i Gènere a la Universitat Jaume l, Castelló: Universitat Jaume I. Disponible en: https://documents.uji.es/alfresco/d/d/workspace/SpacesStore/b9ba333f-748b-44a5a797-1559c8395768/9788417429065.pdf? guest=true (Fecha de consulta: 12/2/19). 
Iversen, Eva y Regis Nyamakanga (eds.) (2012). Because I am a Girl. Africa report 2012. Progress and Obstacles to Girl's Education in Africa. Disponible en: https://planinternational.org/publications/progress-and-obstacles-girls-education-africa (Fecha de consulta: 12/2/19).

NACIONES UNIDAS (2015). Educación de calidad: por qué es importante. Disponible en: https:// www.un.org/sustainabledevelopment/es/wp-content/uploads/sites/3/2016/10/4_ Spanish_Why_it_Matters.pdf (Fecha de consulta: 12/2/19).

ORTEGA, Delfín (2018). «Las mujeres en la historia enseñada: resultados de un programa docente en formación inicial del profesorado» en Enseñanza de las Ciencias Sociales, №17, pp. 13-21.

RAMIRO, Enric (2014). «Química de gènere des de la didáctica de les ciències socials. Una visió sobre la igualtat de gèneres a través de la historia de la química i un recurs per desenvolupar» en Comunicació educativa, №27, pp.40-46.

Ramiro, Enric; Selma, Sergi y Sara Prades (2015). «Els jocs de la pizza. Una proposta intercultural des de la didáctica de les Ciències Socials» en Nos, Eloísa et al. (eds.) (2015). \#comunicambio: Comunicación y Sociedad Civil para el Cambio, Madrid: Fragua, pp. 1029-1039.

RAMIRO, Enric y José Vicente GIL (2017). «Imaginació i creativitat matemàtica a les Ciències Socials». @tic. Revista d'innovació educativa, N¹9, pp.56-61.

RAmIRO, Enric (2017). «Las matemáticas nos alegrarán la cara» en Uno. Revista de Didáctica de las matemáticas, №76, pp. 51-56.

Ramiro, Enric y Santos Ramírez (2018). «Juego didáctico: la baraja de África» en Iber: didáctica de las ciencias sociales, geografía e historia, N92, pp.73-75.

Ruzz, Xuxo (2013). Educando con magia. El ilusionismo como recurso didáctico, Madrid: Narcea. Sherard, Wade (1998). Mathemagic in the Classroom, Portland: Walch Publishing.

VAlCÁrCEL, Amelia (2017). «La agenda sobrevenida del feminismo» en El País, 17 de junio de 2017. Disponible en:

https://elpais.com/elpais/2017/05/25/opinion/1495703140_313473.html (Fecha de consulta: $15 / 1 / 19)$. 